\title{
The SuperK-gadolinium project
}

\author{
Luis Labarga ${ }^{* t}$ \\ University Autonoma Madrid \\ E-mail: luis.labarga@uam.es \\ on behalf of The Super-Kamiokande Collaboration
}

On June 2015, the Super-Kamiokande (SK) Collaboration approved the SuperK-Gadolinium project that, by dissolving a Gd salt at $0.2 \%$ in mass in the SK water, will upgrade the detector to be able to identify neutrons with very high efficiency. The current expected time for refurbishment of SK and start of this new phase is 2018.

In this talk we present the physics benefits of high efficiency neutron tagging in SuperKamiokande, the very extensive R\&D program followed towards the decision, and the most relevant steps in the implementation of the SuperK-gadolinium project.

XIII International Conference on Heavy Quarks and Leptons

22-27 May, 2016

Blacksburg, Virginia, USA

\footnotetext{
* Speaker.

$\dagger$ supported by EU H2020-MSCA-RISE-2014-GA641540, SKPLUS.
} 


\section{Introduction}

The Super-Kamiokande experiment (Fig. 1) is currently the world's most powerful scientific apparatus for nucleon decay and neutrino physics. SK discovered atmospheric neutrino oscillations, it was key in the solution of the solar neutrino problem and it is measuring precisely an important fraction of the elements of the leptonic mixing matrix [1]. SK, operating as KEK-toKamioka (K2K) far detector, observed neutrino oscillations in a long baseline experiment for the first time and measured precisely its characteristics. SK, operating as Tokai-to-Kamioka (T2K) far detector, has provided evidence of a non-vanishing value for $\theta_{13}$, opening the door to the discovery of $\mathrm{CP}$ violation in the lepton sector [2].

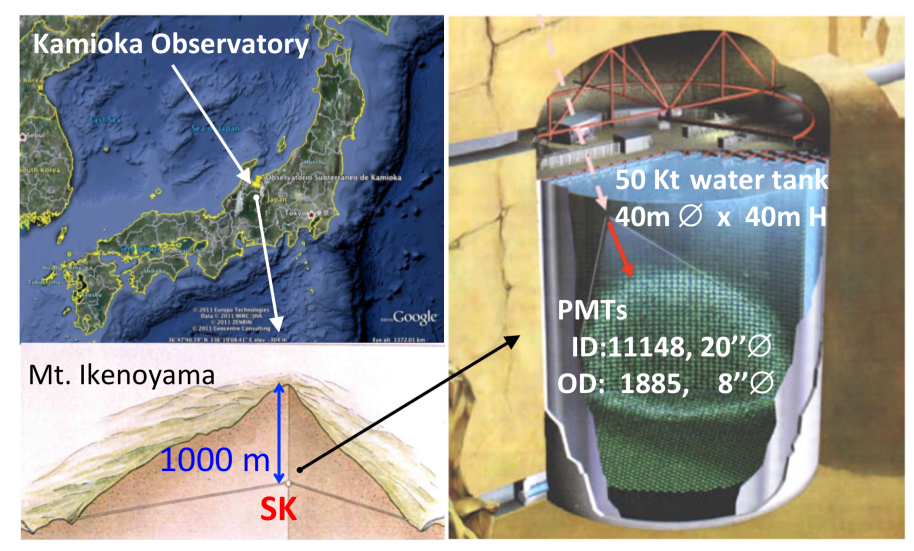

Figure 1: The Super-Kamiokande experiment, under Mount Ikenoyama, in the Kamioka Observatory (Kamioka, Gifu prefecture, Japan). It is divided into an inner detector (ID, 32 kton of water) surrounded by the outer detector (OD, 18 kton).

Concerning proton-decay searches, SK is providing the current world's best limits for the most relevant modes [3]. On the astrophysics side, SK and its precursor Kamiokande, pioneered the field of neutrino astronomy, and is currently providing many of the world's best limits on various astrophysical objects paradigmatically Dark Matter (DM) and Supernova Relic Neutrinos [4].

From the instrumentation point of view, the success of SK comes from the water-Cherenkov technique, which allows to instrument huge amounts of active mass with a relatively simple, reliable, understood and low cost technology [6]. However, it has a major drawback: its inability to distinguish neutrinos from antineutrinos, this issue being at the heart of experimental $v$ physics.

In this context Beacon and Vagins propossed [7] the addition to the SK water of a Gadolinium solute. The Gd has a very large cross-section for thermal neutron capture that is accompanied by the release of energetic $\gamma$ s, thus allowing SK a very efficient ( $\approx 90 \%$ capture, $\approx 90 \%$ reconstruction) tagging of neutrons. This ability opens Super-Kamiokande to a whelm of processes of major scientific interest that will be discussed in Section 2.

The SK Collaboration did setup a strong R\& D program (named originally GADZOOKS!) in this direction. Its "proof-of-principle" was published by Super-Kamiokande in [8]. In section 3 the key elements and conclusions of this $\mathrm{R} \& \mathrm{D}$ will be presented. Also in this section we will describe our works and conclusions on the issue of the radioactive contamination brought mainly by the $\mathrm{Gd}$ 
salt, and its impact on the Superk-Gadolinium physics. As a result: we will add $\approx 100$ tons of the water-soluble gadolinium compound $\mathrm{Gd}_{2}\left(\mathrm{SO}_{4}\right)_{3}$ to the Super-Kamiokande ultra-pure water. The status of the project and its main milestones will be detailed in Section 4.

\section{Physics Benefits}

Very high efficiency neutron tagging will be key for the expected discovery by Super-Kamiokande of the Diffuse Supernova Neutrino Background (DSNB) also known as Supernova Relic Neutrino (SRN). Fig. 2-left shows the energy dependence of the measured flux of low energy electron neutrinos by SK along a sample o reasonable predictions for the corresponding spectrum of SRN. Clearly, the background (mainly from spallation) and the solar flux itself, is above the DSNB expectation by many orders of magnitude.

The ability of unambiguously identifying inverse $\beta$ reactions of anti- $v$ on proton $\left(\bar{v}_{e} p \rightarrow e^{+} n\right)$ from the coincidence in time (within tens of $\mu \mathrm{s}$ ) and space (within tens of $\mathrm{cm}$ ) between the Cherenkov lights of the $e^{+}$(or the anti-lepton corresponding to the flavor of the incoming $v$ ) and the $e^{-}$,s Compton scattered from the $\gamma, \mathrm{s}$ released by the Gd (Fig. 2-middle), allows SK-Gd to reduce dramatically the background to levels, already similar to current expectations for SRN [5] (Fig. 2-left). Fig. 3 shows the expected number of recorded events for those models during 10 years of observation.
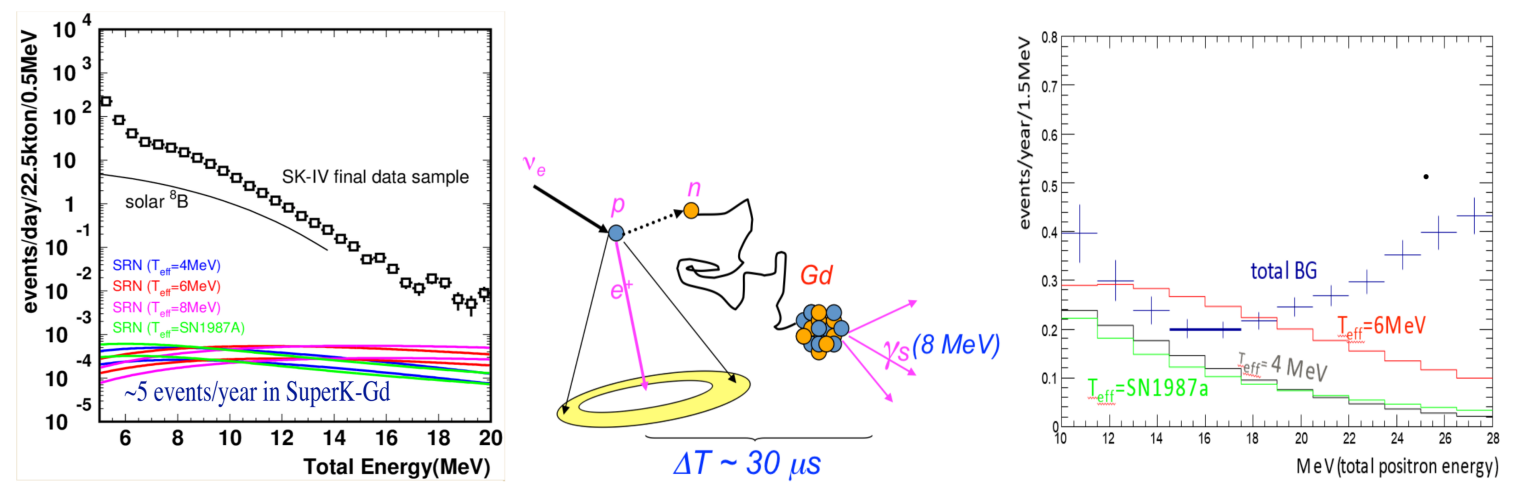

Figure 2: Left: measured energy dependence of the low energy electron neutrino flux by SK along a sample o reasonable predictions for the corresponding SRN spectrum. Middle: sketch of the inverse $\beta$ reaction and its identification by SK-Gd. Right: expectations for the measured spectrum of $\bar{v}$ by Superk-Gd along estimations from contribution from SRN $\bar{v}$.

In the event of a nearby supernova it would be most beneficial to have $\mathrm{Gd}_{2}\left(\mathrm{SO}_{4}\right)_{3}$ in SuperKamiokande. This is primarily because the most copious supernova neutrino signal by far $(\sim 88 \%)$ in water comes from inverse $\beta$ events. They are only produced by one of the six species of neutrinos and antineutrinos which are generated by a stellar collapse, and so if we could tag them individually by their follow-on neutron captures then we could extract the $\bar{v}_{e}$ time structure of the burst precisely, gaining valuable insight into the dynamics of the burst. Also, they can be subtracted away from the more subtle non- $\bar{v}_{e}$ signals, uncovering additional information that would otherwise be lost.

If the exploding star was big and rather close ( $\sim 1 \mathrm{kpc}$ or less), we could get an early warning of its impending collapse [9]. Approximately a week before exploding, the turn-on of Si fusion 


\begin{tabular}{|l|c|c|c|c|}
\hline model & $\begin{array}{c}10-16 \mathrm{MeV} \\
\text { (evts/10yrs) }\end{array}$ & $\begin{array}{c}16-28 \mathrm{MeV} \\
\text { (evts/10yrs) }\end{array}$ & $\begin{array}{c}\text { Total } \\
\text { (10-28MeV) }\end{array}$ & $\begin{array}{r}\text { significance } \\
\text { (2 energy bin) }\end{array}$ \\
\hline $\mathrm{T}_{\text {eff }} 8 \mathrm{MeV}$ & 11.3 & 19.9 & 31.2 & $5.3 \sigma$ \\
\hline $\mathrm{T}_{\text {eff }}$ 6MeV & 11.3 & 13.5 & 24.8 & $4.3 \sigma$ \\
\hline $\mathrm{T}_{\text {eff }} 4 \mathrm{MeV}$ & 7.7 & 4.8 & 12.5 & $2.5 \sigma$ \\
\hline $\mathrm{T}_{\text {eff }}$ SN1987a & 5.1 & 6.8 & 11.9 & $2.1 \sigma$ \\
\hline BG & 10 & 24 & 34 & --- \\
\hline
\end{tabular}

Figure 3: Expected number of detected DSNB events by SuperK-Gd with 10 years operation.

in the core would raise the temperature of the star sufficiently that electron-positron annihilations within its volume would begin to produce $v$ s just above inverse $\beta$ threshold. The sub-Cherenkov $\mathrm{e}^{+}$would be invisible, but in SK the captures of the resulting neutrons on $\mathrm{Gd}_{2}\left(\mathrm{SO}_{4}\right)_{3}$ would result in a sudden and continuing increase of our usual SLE singles rate. As early as six days before collapse we would see a 5-sigma excursion in our final solar neutrino sample's low energy event rate.

Inverse $\beta$ interaction identification via Gd opens SK the long-distance detection of reactor $\bar{v}$ with high efficiency. Since their spectrum is rather soft the detection of these positron singles is currently overwhelmed by radioactive backgrounds. Also, the present threshold of Super-Kamiokande is too high: more than two-thirds of the interactions are below threshold. Even after five years of Gd running the $\Delta m^{2}$ constraint is substantially improved. Ten years of running will significantly improve the world's uncertainty of all accessible oscillation parameters.

At higher energies (order of $\mathrm{GeV}$ ) antineutrinos are more likely to produce a neutron in the primary interaction than neutrinos, basically for all type of reactions. Thus the number of neutrons detected after a neutrino interaction is expected to follow this tendency. Despite of the fact that the neutrons produced in the final state increase with increasing energy, the result of interactions inside the nucleus, However at energies particularly sensitive to CP violation effects (around 0.5 $\mathrm{GeV}$ ) and Mass Hierarchy (few $\mathrm{GeV}$ ) their differences are significant. As illustration Fig. 4-left shows the multiplicities of tagged neutrons in $0.5 \mathrm{GeV}$ to $0.7 \mathrm{GeV}$ neutrino and anti-neutrino interactions. Neutron multiplicities are also expected to differ in $\mathrm{NC}$ and $\mathrm{CC}$ atmospheric neutrino interaction final states. Fig. 4-middle shows the expectations for neutrino energies above $1 \mathrm{GeV}$ in reconstructed Multi-ring e-like events.

We estimate that the implementation of those, Gd driven, neutron multiplicity differences in the full oscillation analysis of atmospheric neutrinos, will improve its sensitivity to the neutrino Mass Hierarchy by $\sim 50 \%$ and $\sim$ double that to $\delta_{C P}$ (Fig. 4-right).

High efficiency neutron tagging improves significantly the sensitivity of proton decay searches. In general, less neutrons should be emitted after genuine proton decays than in background processes caused by atmospheric neutrino interactions. Fig. 5-left shows the number of neutrons expected after proton decays via $p \rightarrow e^{+} \pi^{0}$. The requirement of no associated neutron to the events surviving the final selection cuts used in the current analysis, decreases the detection efficiency only by $6 \%$. On the other hand, the overwhelming majority of background events arising from 

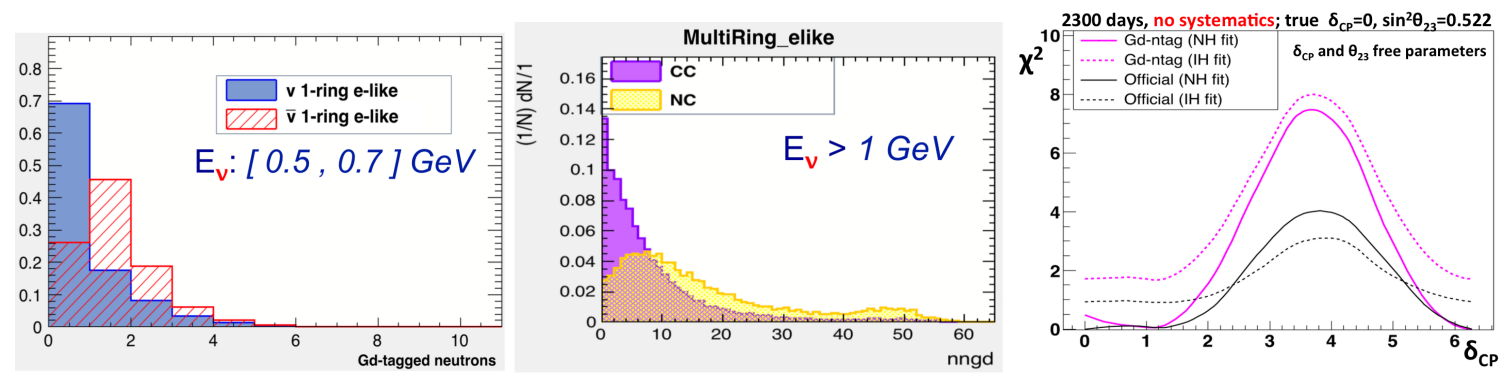

Figure 4: Left: neutron multiplicity distributions for $0.5 \mathrm{GeV}$ to $0.7 \mathrm{GeV}$ one ring e-like sample split into neutrinos (blue) and antineutrinos (red) and normalized to unity. Middle: neutron multiplicity distributions for NC and CC atmospheric neutrino interactions. Right: Sensitivity to $\delta_{C P}$ in the cases of Normal and Inverted Hierarchies, for the current SK analysis and assuming Gd neutron tagging.

atmospheric neutrino interactions have one or more neutrons in their final states (Fig. 5-right). The estimated reduction of the atmospheric neutrino background by neutron tagging (veto) with $\mathrm{Gd}$ is $\approx 83 \%$. Therefore a significant improvement on the sensitivity will be obtained. However, the benefit from Gd-enabled neutron tagging is even greater when one candidate is observed. For example, after 10 years of observation the expected background with the current analysis is 0.58 events, while it will be reduced to 0.098 events with Gd. Poisson probabilities to observe one or more events with these expected backgrounds are $44 \%$ and $9 \%$ respectively. I.e. we can claim an indication of proton decay based upon even a single candidate event with more than 90\% C.L..
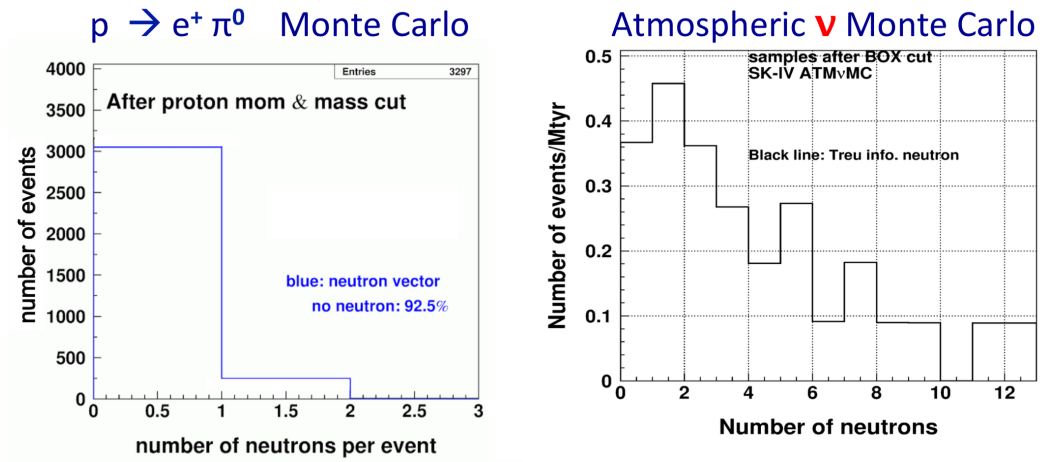

Figure 5: Neutron multiplicity in proton decay MC (left) and atmospheric MC (right).

\section{The EGADS demonstrator}

The EGADS [Evaluating Gadolinium's Action on Detector Systems] facility was built in the Kamioka mine explicitly to demonstrate that the gadolinium loading of Super-Kamiokande would be safe and effective. Its key elements are depicted in Fig. 6. Thet are a 200-ton water Cherenkov detector instrumented with the same 20-inch PMTs as those used in SK, a pre-treatment system with a 15-ton tank for dissolving and mixing $\mathrm{Gd}_{2}\left(\mathrm{SO}_{4}\right)_{3}$ into the water, the main $\mathrm{Gd}$-capable water circulation system, and a water transparency automatic measurement device (UDEAL). 


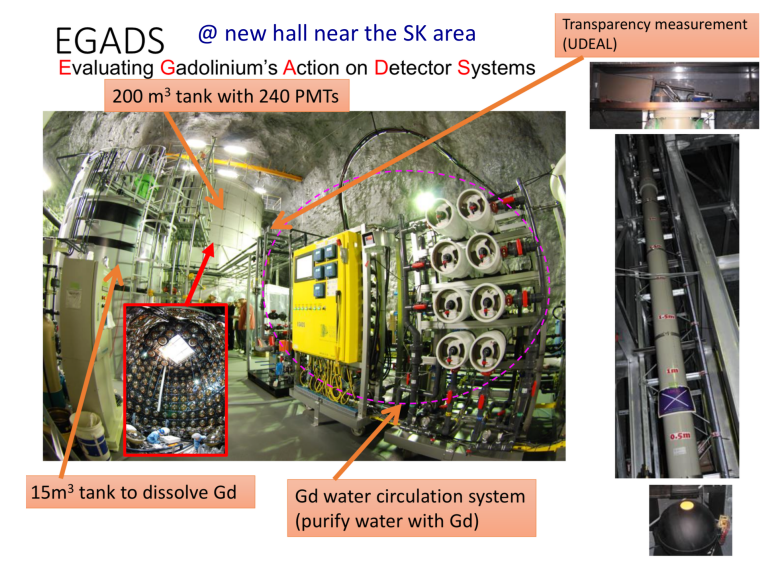

Figure 6: A schematic view of the EGADS facility.

The light attenuation length in water must be kept long to meet the various physics goals in Super-Kamiokande even if Gd is loaded. The main EGADS water system must be capable of performing selective filtration, since the desirable multivalent ions $\mathrm{Gd}^{3+}$ and $\left(\mathrm{SO}_{4}\right)^{2-}$ must be retained in solution while all other particles, gasses, and other contaminants must be removed. A new technology called "ionic band-pass filtration" has been developed for this very purpose [10]. A schematic view of this system is shown in Fig. 7.
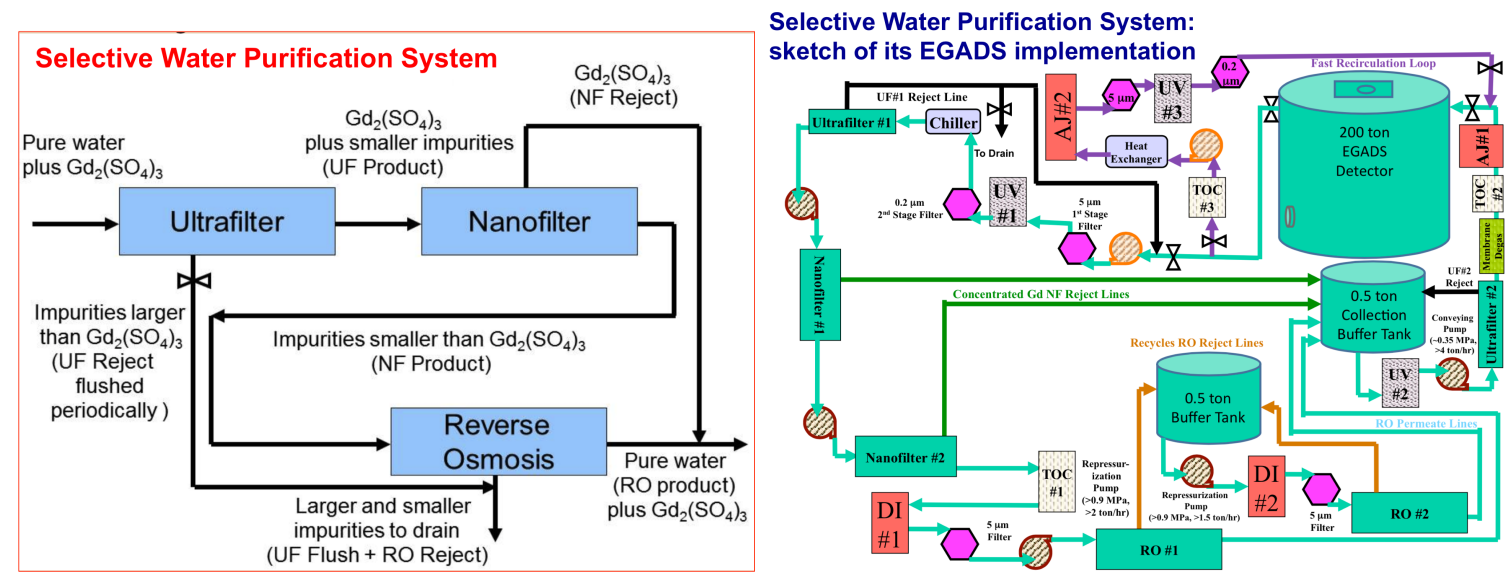

Figure 7: Left: working scheme of the "ionic band-pass filtration" technology. Right: Schematic view of the EGADS main water circulation system.

After being pre-cooled in a chiller to prevent precipitation of the gadolinium sulfate (less soluble as temperature increases) the Gd-loaded water from the 200-ton EGADS detector is sent through micro-filtration and ultrafiltration. The selective filtration occurs at the next step, nanofiltration. The membrane pore size of certain nano-filters (NF) is smaller than the $\mathrm{Gd}^{3+}$ and $\left(\mathrm{SO}_{4}\right)^{2-}$ ions, so they are selected and placed in the NF reject stream. The Gd-free water which passes through the NF is then sent through deionization (DI) and reverse osmosis (RO) stages, which only $\mathrm{H}_{2} \mathrm{O}$ molecules can pass. The resulting ultra-pure water and the $\mathrm{Gd}_{2}\left(\mathrm{SO}_{4}\right)_{3}$ are reunited 
in the collection buffer tank and sent back to the 200-ton detector.

One of the main goals of the EGADS project is to compare the water transparency of ultra-pure water to that of water doped with the concentration of gadolinium sulfate needed for the Super-KGd experiment, and determine if the light transmission properties are enough to both, carry out new physics program and continue with the physics studies currently under way at SK. The transparency of the water inside the EGADS detector is measured and monitored using the custom built UDEAL device (right-hand side of Fig. 6). It measures the absolute attenuation length at seven different wavelengths (their contribution to Cherenkov light is indicated in brackets): $337 \mathrm{~nm}(0.25), 375$ $\mathrm{nm}(0.25), 405 \mathrm{~nm}(0.21), 445 \mathrm{~nm}(0.14), 473 \mathrm{~nm}(0.11), 532 \mathrm{~nm}(0.04), 595 \mathrm{~nm}(.003)$. A very useful variable for the purpose is LL15: the percentage of Cherenkov light that would be left after $15 \mathrm{~m}$ (the characteristic photon travel distance inside the SK detector). The "measured" Cherenkov light is taken as the weighted sum of the amount of light left at each wavelength, with the weights given by the above fractions of Cherenkov light represented by each of the measured wavelengths.
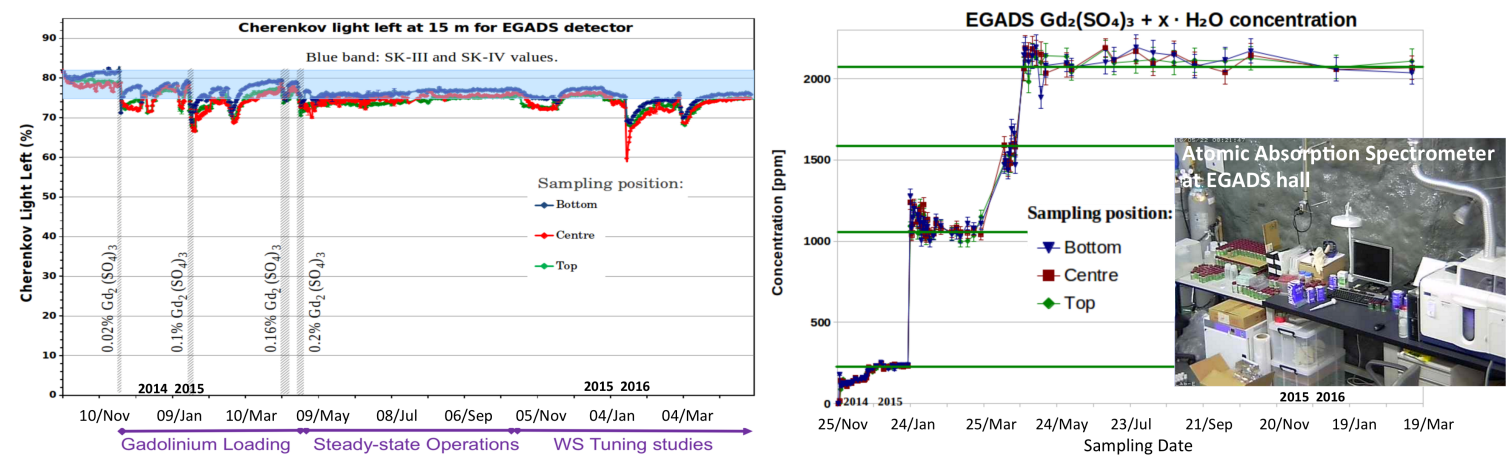

Figure 8: Left: Percentage of Cherenkov light remaining for water inside the instrumented 200-ton EGADS tank for increasing concentrations of gadolinium sulfate (injection periods are indicated by vertical bands). The green, red, and blue lines correspond to data taken from the top, center, and bottom sample points of the 200-ton tank, respectively, while the light blue band shows the range of ultra-pure water transparencies during SK-III and SK-IV. Right: Gadolinium sulfate concentration history, as measured by the AAS, for the three sampling points in the top, center and bottom of the EGADS detector.

Fig. 8-left shows the history of the measured LL15 since October $11^{\text {th }}, 2014$. The four loadings are clearly identified. It demonstrates that 1) transparencies can be comparable to those attained at the pure-water SK's phases III and IV,2) the period of recovery after any type of intervention (Gd loading, water-system tuning, etc.) is rather short, and 3) in steady operation the measured transparencies are nicely stable at values very appropriate for physics analyses.

The gadolinium sulfate concentration in the 200-ton tank is monitored by using an Atomic Absorption Spectrometer (AAS). Water samples are collected by hand from three points in the detector, at three different heigh, using the same pipe as the UDEAL sampling. The uncertainty of the measurement is around 3.5\%. Fig. 8-right shows the measured concentration since November $27^{\text {th }}, 2014$. The four loadings are clearly seen as steps in the concentration. The concentrations at all three sampling points are: 1) very close to each other, indicating that there is a homogeneous solution in the 200-ton tank, and 2) stable between loadings or any other external intervention, demonstrating that there is no significant Gd loss during continuous water recirculation and purification. 

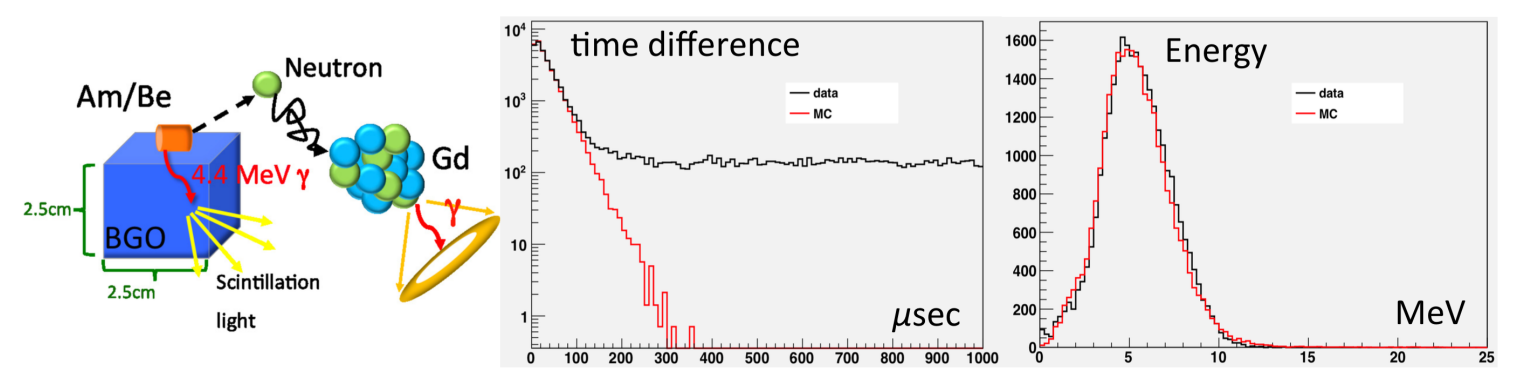

Figure 9: Left: Schematic view of how the Am/Be source and BGO scintillator mimics the inverse $\beta$ signal. Middle, Right: Time difference distribution (middle) and energy distribution (right) of the data and MC for $2178 \pm 44 \mathrm{ppm} \mathrm{Gd}_{2}\left(\mathrm{SO}_{4}\right)_{3}$.

The demonstration of neutron capture by gadolinium-loaded water and evaluation of its efficiency is done using a Am/Be radioactive source, imbedded in a BGO crystal. This system mimics the inverse $\beta$ signal(see Fig. 9-left). It is deployed inside the EGADS detector. The relevan reaction is ${ }^{241} \mathrm{Am} \rightarrow{ }^{237} \mathrm{~Np}+\alpha ; \alpha+{ }^{9} \mathrm{Be} \rightarrow{ }^{12} \mathrm{C}^{*}+\mathrm{n},{ }^{12} \mathrm{C}+\mathrm{n} ;{ }^{12} \mathrm{C}^{*} \rightarrow{ }^{12} \mathrm{C}+\gamma(4.43 \mathrm{MeV})$ with the BGO emmiting scintillation light when hit by $\gamma$-rays.

$\mathrm{Am} / \mathrm{Be}$ data were taken at most of the steps of $\mathrm{Gd}_{2}\left(\mathrm{SO}_{4}\right)_{3}$ loading i.e., at $0.02 \%, 0.1 \%$, and the full loading of $0.2 \%$. The middle panel of Fig. 9 shows the time difference from the prompt event to the neutron capture at $0.2 \%$ loading. Data and MC are in good agreement for the mean capture time in the three concentrations. The plot on the right-most side of Fig. 9 shows the energy distributions of $\mathrm{Gd} \gamma$-ray candidates for data and $\mathrm{MC}$ at $0.2 \%$ loading. They are also in good agreement, peaking around the expected $5 \mathrm{MeV}$.

Another important line of research, not directly related to EGADS, is the potential impacts of adding Gd to SK due to its possible radioactive contamination. Several batches of the compound that will be used for loading SK, $\mathrm{Gd}_{2}\left(\mathrm{SO}_{4}\right)_{3}$, have been bought from different companies in order to measure and compare their radioactive contaminants. These measurements have been performed at Canfranc Underground Laboratory (LSC) in Spain, and are summarized in Fig. 10. It lists the company and date from which each batch was ordered, with the measured radioactive impurities split into different radioactive decay chains and other isotopes:

\begin{tabular}{|c|c|c|c|c|c|c|c|c|c|c|}
\hline Chain & $\begin{array}{l}\text { Sub- } \\
\text { chain }\end{array}$ & $\begin{array}{c}\text { Standford } \\
\text { Materials } \\
09 / 04\end{array}$ & $\begin{array}{c}\text { Standford } \\
\text { Materials } \\
10 / 08\end{array}$ & $\begin{array}{c}\text { Beijing } \\
\text { Jinghonganxin } \\
12 / 08 \\
\end{array}$ & $\begin{array}{c}\text { Changshu } \\
\text { Huanyu } \\
13 / 02\end{array}$ & \begin{tabular}{|c|} 
Beijing \\
Jinghonganxin \\
$13 / 03$ \\
\end{tabular} & $\begin{array}{c}\text { Standford } \\
\text { Materials } \\
13 / 08\end{array}$ & $\begin{array}{c}\text { HK Tai } \\
\text { Kun } \\
13 / 07 \mathrm{a}\end{array}$ & $\begin{array}{c}\text { HK Tai } \\
\text { Kun } \\
13 / 07 \mathrm{~b}\end{array}$ & $\begin{array}{c}\text { Standford } \\
\text { Materials } \\
14 / 12\end{array}$ \\
\hline \multirow{2}{*}{${ }^{238} \mathbf{U}$} & ${ }^{238} \mathrm{U}$ & $51 \pm 21$ & $<33$ & $292 \pm 6$ & $74 \pm 28$ & $242 \pm 6$ & $71 \pm 20$ & $47 \pm 26$ & $73 \pm 27$ & $<76$ \\
\hline & ${ }^{226} \mathbf{R a}$ & $8 \pm 1$ & $2.8 \pm 0.6$ & $74 \pm 2$ & $13 \pm 1$ & $13 \pm 2$ & $8 \pm 1$ & $5 \pm 1$ & $6 \pm 1$ & $<1.4$ \\
\hline \multirow{2}{*}{${ }^{232} \mathrm{Th}$} & ${ }^{228} \mathbf{R a}$ & $11 \pm 2$ & $270 \pm 16$ & $1099 \pm 12$ & $205 \pm 6$ & $21 \pm 3$ & $6 \pm 1$ & $14 \pm 2$ & $3 \pm 1$ & $2 \pm 1$ \\
\hline & ${ }^{228} \mathbf{T h}$ & $28 \pm 3$ & $86 \pm 5$ & $504 \pm 6$ & $127 \pm 3$ & $374 \pm 6$ & $159 \pm 3$ & $13 \pm 1$ & $411 \pm 5$ & $29 \pm 2$ \\
\hline \multirow{2}{*}{${ }^{235} \mathbf{U}$} & ${ }^{235} \mathrm{U}$ & $<32$ & $<<32$ & $<112$ & $<25$ & $<25$ & $<<32$ & $<12$ & $<30$ & $<1.8$ \\
\hline & ${ }^{227} \mathbf{A c}$ & $214 \pm 10$ & $1700 \pm 20$ & $2956 \pm 30$ & $1423 \pm 21$ & $175 \pm 42$ & $295 \pm 10$ & $<6$ & $<18$ & $190 \pm 6$ \\
\hline \multirow{3}{*}{ Others } & ${ }^{40} \mathbf{K}$ & $29 \pm 5$ & $12 \pm 3$ & $101 \pm 10$ & $60 \pm 7$ & $18 \pm 8$ & $3 \pm 2$ & $3 \pm 2$ & $8 \pm 4$ & $<5$ \\
\hline & ${ }^{138} \mathbf{L a}$ & $8 \pm 1$ & $<$ & $683 \pm 15$ & $3 \pm 1$ & $42 \pm 3$ & $5 \pm 1$ & $<1$ & $<2$ & $23 \pm 1$ \\
\hline & ${ }^{176} \mathrm{Lu}$ & $80 \pm 8$ & $21 \pm 2$ & $566 \pm 6$ & $12 \pm 1$ & $8 \pm 2$ & $30 \pm 1$ & $1.6 \pm 0.3$ & $<2$ & $2.5 \pm 0.6$ \\
\hline
\end{tabular}

Figure 10: Radioactivity in various batches of $\mathrm{Gd}_{2}\left(\mathrm{SO}_{4}\right)_{3}$ as measured at the LSC.

These measurements indicate the presence of non-negligible radioactive contamination in the 
Gd salt. Further, the radioactive chains do not appear to be in equilibrium. These levels will be monitored, and reduced where necessary, to maintain control of backgrounds. All incoming batches of gadolinium sulfate will be surveyed and batches with excess contamination will not be allowed into SK.

We have estimated the impact of typical levels of contaminations on SK's low energy performance. The potential backgrounds for SRN arise mainly from ${ }^{238} \mathrm{U}$ spontaneous fission, as well as accidental coincidences due to neutrons and beta decays. It was found to be several counts/FV/year which is similar as the expected SRN signal rate. However, it has been determined that the pretreatment of the dissolved gadolinium by Amberjet 4400 reduces the uranium levels by another factor of $\sim 200$, thus not jeopardizing the SRN measurement

Early supernova warning: the handful of inverse $\beta$ events expected in Super-K during Si burning for any pre-supernova candidate star will occur at a much higher rate (on the order of one per hour) than the usual SRN signal (on the order of one every two months). Therefore, the supernova early warning analysis will, like the SRN analysis, not be adversely affected by radioactive contaminants in the $\mathrm{Gd}_{2}\left(\mathrm{SO}_{4}\right)_{3}$.

The lower energy bins in the solar analysis (current threshold is $3.5 \mathrm{MeV}$ recoil electron kinetic energy) could be heavily contaminated with $\gamma \mathrm{s}$ coming from radioactive radium daughters, primarily ${ }^{208} \mathrm{Tl},{ }^{212} \mathrm{Bi}$, and ${ }^{214} \mathrm{Bi}$. They would become the main source of solar neutrino background and could be expected to be three orders of magnitude larger than the solar neutrino signal. Therefore, a further purification method should be applied to the Gd-loaded water in order to reduce - by at least that factor - the amount of ${ }^{228} \mathrm{Th} /{ }^{228} \mathrm{Ra}$ and ${ }^{238 U} / 226 \mathrm{Ra}$ present in the Gd salt. Work to achieve this reduction is ongoing.

In the last months we have started cooperation with several new companies specialized in high purity rear earth products. They are Molycorp, Shin-Etsu Chemical Co. Ltd. , Kanto Chemical Co. Inc., Wako Pure Chemical Inc. Ltd., and Nippon Ytrium Co. Ltd.. They have provided samples that we have measured obtaining very promising results. The Kamioka Observatory and the Boulby Underground Observatory have recently joined the LSC in our works of measuring radioactive contaminations.

\section{Implementation in Super-Kamiokande}

A T2K+SK joint protocol is being stablished to take the decision about when to trigger the project and its implementation. It takes into account the needs of both experiments, the readiness of SK-Gd project, the T2K schedule, the J-PARC MR power upgrade and other miscellanea elements. The current expected time of start is 2018. Respecting the SK detector probably the most critical items are the new water system and its refurbishment (mainly stop the leak).

The scaling up of EGADS for SuperK-Gd new water system is well under way. There is already a detailed design of a highly compact, cost-saving system. Two companies will be competing for its design: ORGANO (Japan) and South Coastal Waters (USA). The system will be hosted in a new-built large experimental hall $\left(\approx 4 \mathrm{k} \mathrm{m}^{3}\right)$ located near the SK area. This hall has been constructed from the start to contain fluids and not release them into the mine. This is accomplished via waterproof coating on the floors and walls and high-quality, water-tight doors leading to exterior 
areas. Finally, sump pumps will be in place to direct any spilled water through ion-exchange resin tanks. The hole is fully ready for occupancy.

The propossed procedure for stopping the water leak in the Super-K tank - or at least reducing it to a negligible amount - involves covering all the submerged welded areas on the surface of the SK tank with sealing materials. The approach we are following is to employ two sealants with very different mechanical properties. The first one is designed to sneak into small gaps and push entrained water out of the way. Our best candidate for the job is BIO-SEAL 197, originally developed by Thin Film Technology, Inc., to repair leaks in the pressure housings of nuclear submarines while they were deployed at sea.

BIO-SEAL is designed to be strong but it is rather rigid. Since we are concerned about the SK tank potentially deforming when it is filled and drained, we plan to cover the BIO-SEAL with another material with no crack-seeking ability but possessing greater elasticity to allow for more displacement. The candidate for this overcoating sealant is MineGuard. It is routinely used in the Kamioka Observatory and it was also used to form the outer tank of the SNO detector. Of the different formulations available our candidate one uses poly-urea because it does not suffers from hydrolysis and shows very low radon emanation. We are conducting regular meetings with Mitsui Engineering regarding the exact resin application procedure to be employed inside the SK tank.

The current plan for the implementation of Superk-Gd has three phases. The first period is for the leak repair work, pure water fill, and recirculation of pure water in the detector until good water transparency is achieved. So, counting from the beginning of this period it takes $\approx 6$ months until the tank becomes full again, as well as a few additional months after that while the water transparency recovers. The second period is for loading $\mathrm{Gd}_{2}\left(\mathrm{SO}_{4}\right)_{3}$ up to $0.02 \%$, which corresponds to $\approx 50 \%$ neutron capture efficiency on Gd. Including a brief pause at $0.002 \%$, the loading itself would take about two months, followed by an additional few months of water quality stabilization. We will run the detector for a while at this concentration in order to study backgrounds and neutron capture efficiency. As shown in Fig. 8 the water transparency at this concentration is within the "SK pure water" range and negative effects on other physics, if any, will be minimal. In the third period we will add the remaining $\mathrm{Gd}_{2}\left(\mathrm{SO}_{4}\right)_{3}$ needed to achieve our full target loading, $0.2 \%$.

\section{References}

[1] Super-Kamiokande Collab.; Y. Fukuda et al., Evidence for oscillation of atmospheric neutrinos, Phys. Rev. Lett. 81 (1989) 1562.

Super-Kamiokande Collab. Y. Ashie et al., Measurement of atmospheric neutrino oscillation parameters by Super-Kamiokande I, Phys. Rev. D71 (2005) 112005.

Super-Kamiokande Collab.; S. Fukuda et al., Determination of solar neutrino oscillation parameters using 1496 days of SK I data, Phys. Lett. B539 (2002) 179.

Super-Kamiokande Collab.; K. Abe et al., Solar neutrino results in Super-Kamiokande-III, Phys. Rev. D83 (2011) 052010.

Super-Kamiokande Collab.; K. Abe et al., A Measurement of the Appearance of Atmospheric Tau Neutrinos by Super-Kamiokande, Phys. Rev. Lett. 110 (2013) 181802.

[2] K2K Collaboration, M.H. Ahn et al., Measurement of Neutrino Oscillation by the K2K Experiment, Phys. Rev. D74 (2006) 072003. 
T2K Collaboration, K. Abe et al., Observation of Electron Neutrino Appearance in a Muon Neutrino Beam, Phys. Rev. Lett. 112 (2014) 061802

[3] Super-Kamiokande Collab.; K. Abe et al., Search for Proton Decay via $p \rightarrow \mu^{+} K^{0}$ in Super-Kamiokande I, II and III, Phys. Rev. D86 (2012) 012006.

Super-Kamiokande Collab.; K. Abe et al., A Search for Nucleon Decay via $n \rightarrow v \pi^{0}$ and $p \rightarrow v \pi^{+}$in Super-Kamiokande, Phys. Rev. Lett. 113 (2014) 121802

Super-Kamiokande Collab.; K. Abe et al., Search for Proton Decay via $p \rightarrow v K^{+}$using 260 kilotonůyear data of Super-Kamiokande, Phys. Rev. D90 (2014) 072005.

[4] Super-Kamiokande Collab.; T. Tanaka et al., An Indirect Search for WIMPs in the Sun using 3109.6 days of upward-going muons in Super-Kamiokande, Astrophys. J. 742 (2011) 78.

Super-Kamiokande Collab.; K. Bays et al., Supernova Relic Neutrino Search at Super-Kamiokande, Phys. Rev. D85 (2012) 052007.

Super-Kamiokande Collab.; H. Zhang et al., Supernova Relic Neutrino Search with Neutron Tagging at Super-Kamiokande IV, Astroparticle Phys. 60 (2014) 41.

[5] S. Horiuchi, J. F. Beacom, E. Dwek, Diffuse supernova neutrino background is detectable in Super-Kamiokande Phys. Rev. D79 (2009) 083013.

[6] Super-Kamiokande Collab.; S. Fukuda et al., The Super-Kamiokande detector, Nucl. Instr. Meth. A 501 (2003) 418.

Calibration of the Super-Kamiokande detector, Nucl. Instr. Meth. A 737 (2014) 253.

[7] J. F. Beacom and M. R. Vagins, Antineutrino spectroscopy with large waterCherenkov detectors, Phys. Rev. Lett. 93 (2004) 171101.

[8] Super-Kamiokande Coll., H. Watanabe et al., First study of neutron tagging with a water cherenkov detector, Astroparticle Phys. 31 (2009) 320.

[9] A. Odrzywolek, M. Misiaszek, M. Kutschera, Detection possibility of the pair-annihilation neutrinos from the neutrino-cooled pre-supernova star, Astroparticle Phys. 21 (2004) 303.

[10] M. R. Vagins (Tokyo U., IPMU), Detection of Supernova Neutrinos Nucl. Phys. Proc. Suppl. 229-232 (2012) 303. 